\title{
RED GIANT STARS: FROM MIXED MODES TO ANGULAR MOMENTUM
}

\author{
K. Belkacem ${ }^{1}$
}

\begin{abstract}
Solar-like oscillations are ubiquitous to low-mass stars from the main-sequence to the red-giant branch as demonstrated by the space-borne missions CoRoT and Kepler. Understanding the physical mechanisms governing their amplitudes as well as their behavior along with the star evolution is a prerequisite for interpreting the wealth of seismic data and for inferring stellar internal structure. In this paper, I discuss our current knowledge of mode amplitudes with particular emphasis on non-radial modes in red giants (hereafter mixed modes). Then, I will show how these modes permit to unveil the rotation of the inner-most layers of low-mass stars and how they put stringent constraints on the redistribution of angular momentum.
\end{abstract}

\section{Introduction: Mixed modes as a cornerstone for unveiling the core of red giants}

Low-mass main-sequence stars with an extended external convective region exhibit acoustic standing waves (hereafter $p$-modes). Those modes, with high amplitudes near the surface, mainly probe the external layers of these stars. It thus allows us to perform stellar seismology as was successfully done using the CoRoT (e.g., Grotsch-Noels \& Deheuvels 2016) and Kepler (e.g., Chaplin \& Miglio 2013) observations. In contrast, $g$-modes, whose restoring force is buoyancy, are potentially able to probe the innermost layers of stars. They have relatively low frequencies and very low amplitudes at the stars surface because they are evanescent in the outer convective region (e.g., Belkacem et al. 2009). However, except for the Sun for which the detection remains controversial (e.g., Appourchaux et al. 2010), those modes have not (yet) been detected in solar-like stars.

For evolved stars, the situation is more favourable. Indeed, while initially thought as complex, oscillation spectra of subgiants and redgiants have proven to

${ }^{1}$ LESIA, Observatoire de Paris, CNRS, PSL Research University, Université Pierre et Marie Curie, Université Denis Diderot, 92195 Meudon, France 


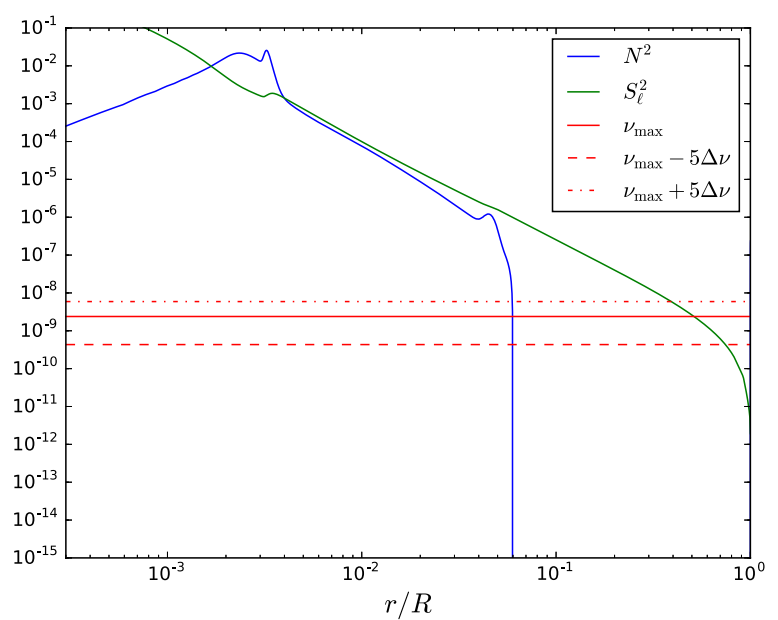

Fig. 1. Propagation diagram showing the squared buoyancy frequency $\left(N^{2}\right)$, the squared Lamb frequency for dipolar modes $\left(S_{\ell=1}^{2}\right)$ versus the normalized radius. The solid red line corresponds to the squared $\nu_{\max }$ frequency computed using the now classical scaling relation (e.g. Belkacem et al. 2011) and the dashed and dashed-dotted red lines correspond to the typical frequency range in which modes are detected. The equilibrium model correspond to an evolved red giant (RGB) stars (and more precisely to model M2 in Belkacem et al. 2015a).

be a rich harvest of information on the innermost layers of these stars. This was made possible thanks to space-borne missions and the first detection by CoRoT of thousands of oscillating redgiant stars (see Mosser \& Miglio 2016, for a review on early results obtained by CoRoT). Those stars exhibit solar-like oscillations with high amplitudes and more importantly non-radial oscillations later identified as mixed modes (e.g., De Ridder et al. 2009). Indeed, a star leaving the mainsequence experiences major structural changes since its radiative core contracts as the result of which its envelope expands. As the total radius increases, both the mean density and the surface gravity decrease. It results in a situation for which a $p$-mode (for a given radial order and angular degree) is found at a lower frequency as does the frequency of the maximum in the power spectrum (as it scales predominantly with the surface gravity, see Belkacem et al. 2011, for details). In contrast, the frequencies of $g$ modes increase because the core contracts while the star evolves. This is due to the increase of the buoyancy frequency. This results in an overlap of the frequency ranges of $p$ and $g$ modes and allow for the appearance of the so-called mixed-modes (e.g., Dziembowski et al. 2001; Dupret et al. 2009). The resonant cavities are depicted by Figure 1 .

These modes have a dual nature because they propagate both in the outer and inner cavities. This is a key advantage because they have amplitude at the surface so that it is possible to detect them and bear information on the innermost regions 
of the star. Obviously, depending on frequency, each mixed mode bears a different information. At first order, it is possible to define and characterize the degree of mixity of a mode by introducing the normalized mode energy, i.e.

$$
E=\int_{0}^{1} \epsilon \mathrm{d} x \quad \text { with } \quad \epsilon=\frac{1}{\xi_{r}(R)^{2}}\left(\xi_{r}^{2}+\Lambda \xi_{h}^{2}\right)
$$

where $\xi_{r}, \xi_{h}$ are the radial and horizontal components for the eigenfunction, $\Lambda=$ $\ell(\ell+1)$ with $\ell$ the angular degree, $x=m / M$, and $M, R$ the total mass and radius respectively. The normalized mode energy given by Equation (1.1) is plotted in Figure 2 (middle panel). For non-radial modes, the normalized mode energy varies with frequency and is found always higher than for radial modes. It allow us to define $p$ and $g$-dominated modes that bear a different information. Indeed, $p$ dominated modes have a low energy and mainly probe the upper layers while the $g$-dominated modes have a high energy and mostly probe the inner-most regions as shown by Figure 2 (bottom panel).

At the time of the first detection and identification of non-radial modes by CoRoT (De Ridder et al. 2009), most of the theoretical background was already developed for decades. These mixed modes, as named in the early works of Dziembowski (1971) for Cepheids and Scuflaire (1974) for a condensed polytropic model, have been subject to an extensive investigation from a theoretical point of view (e.g., Shibahashi 1979; Dziembowski et al. 2001; Christensen-Dalsgaard 2004). Consequently, the foundations being established, the wealth of observations as provided by CoRoT and followed by Kepler gave us the opportunity of a new grip on stellar physics of advanced evolutionary stages.

Nevertheless, a number of fundamental questions were still to be solved. The first crucial issue was the mixed modes detectability and this is addressed in Section 2. Before using those modes for doing seismology and inferring the innermost structure of evolved stars, it was mandatory to be able to identify them and thus to understand the evolution of their amplitude versus the duration of the observations and across the Hertzsprung-Russell diagram. Subsequently, as discussed in Section 3, once mode frequencies are extracted, one must be able to disentangle between several physical effects affecting them such as the glitches, the effect of mode trapping, as well as rotation. Given the complexity of oscillation spectra of evolved stars, this was a crucial prerequisite to be able to properly infer the structure of those stars. Eventually, the mean core rotation of a cohort of evolved stars being inferred, it was possible to emphasize our deficient knowledge about the redistribution of angular momentum in low-mass evolved stars. As we will discuss in Section 4, a number of physical mechanisms are suspected to operate but the picture is far from being grasped and it will certainly give grounds for a harvest of theoretical works in the forthcoming years.

\section{Mixed modes detectability}

For using mixed modes as probes of the internal structure of stars, it is fundamental to understand the physics governing mode amplitudes. Indeed, this knowledge is 

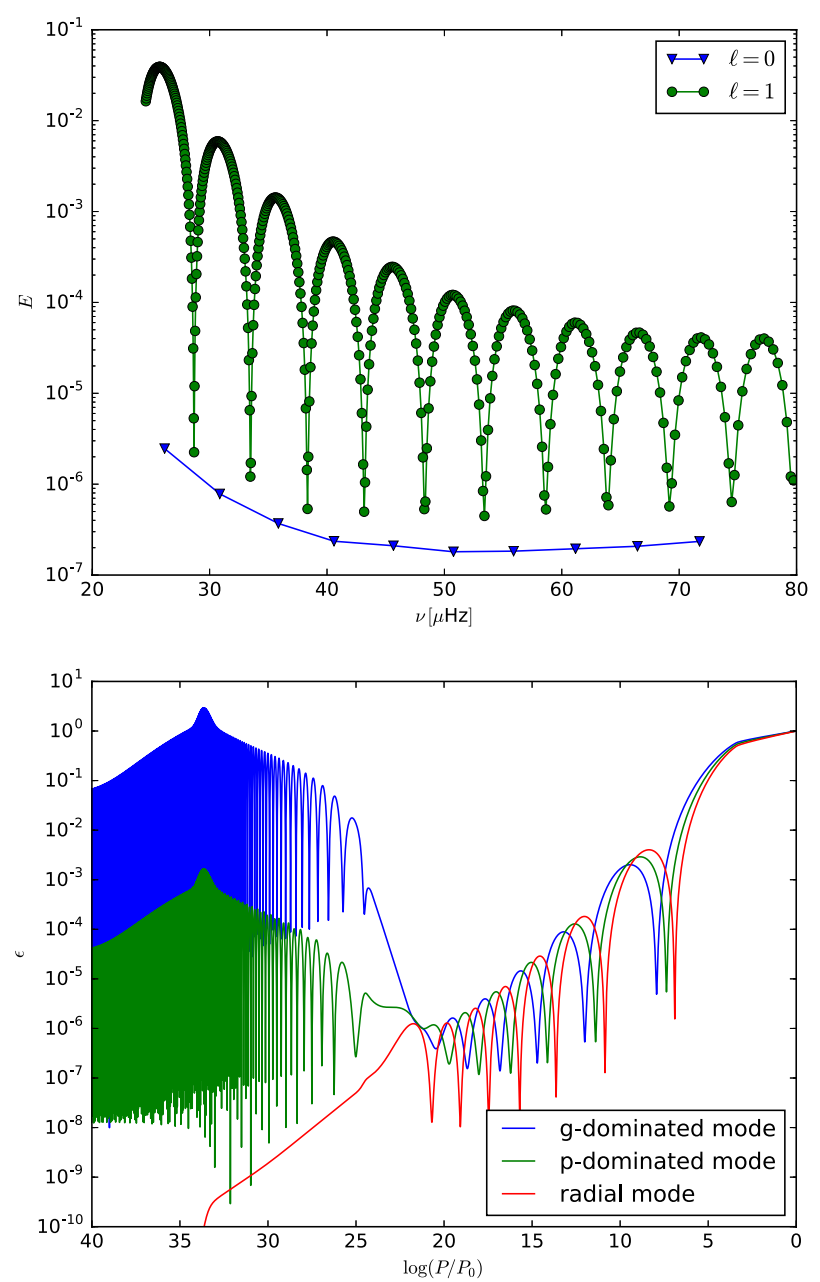

Fig. 2. Top panel: Normalized mode energy computed using Equation (1.1) as a function of the frequency for radial and dipolar modes. Bottom panel: Integrand of the mode energy $\epsilon$ (see Eq. (1.1)) versus the logarithm of the pressure where $P_{0}$ is the pressure at the surface.

mandatory to understand the structure of oscillation spectra. Mode amplitudes in evolved stars is however a long-standing and still not completely solved issue (see the review by Samadi et al. 2015). The problem can be split into two separate subproblems, namely; the amplitude of radial modes and the amplitude of non-radial (or mixed) modes.

\subsection{Radial mode amplitude for evolved stars}

In this section we consider the amplitudes of radial modes in terms of both surface velocity and intensity fluctuations. Based on our knowledge of the solar 


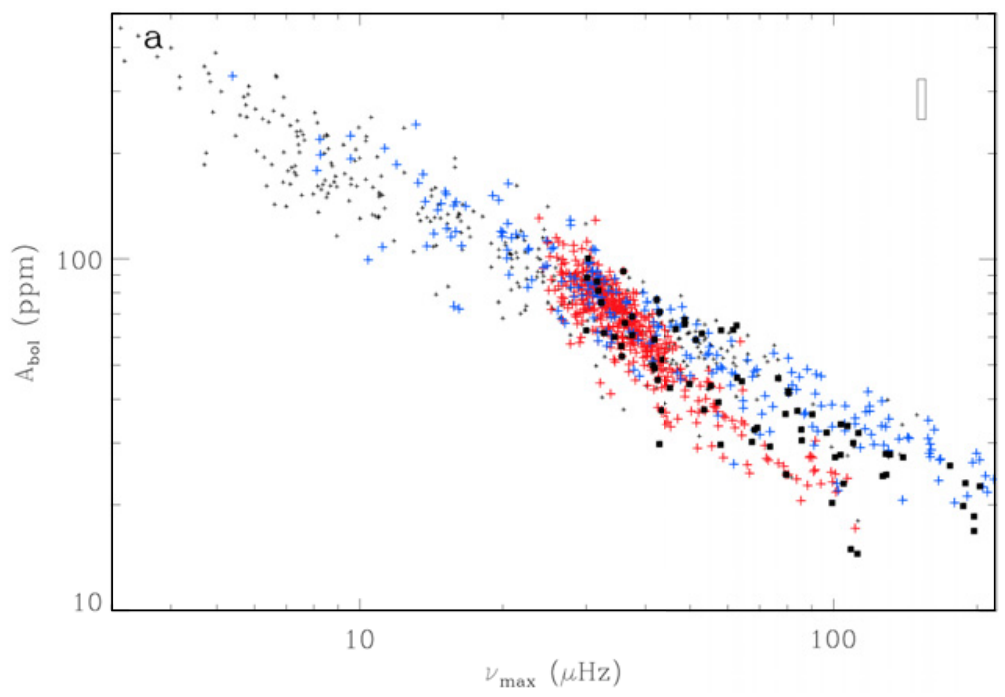

Fig. 3. Maximum height in the power spectrum (at $\nu=\nu_{\max }$ ) for stars observed by Kepler. The color code indicates the evolutionary status; clump stars in red, giant branch stars in blue, unknown status in dark grey. The black squares indicate low-amplitude dipolar modes (see Mosser et al. 2017, for details on those particular stars). From Mosser et al. (2012a).

oscillations, early attempts have been made to extrapolate the calculations to more evolved stars (e.g., Christensen-Dalsgaard \& Frandsen 1983; Kjeldsen \& Bedding 1995; Houdek et al. 1999; Samadi et al. 2007). However, as shown by the space-borne mission (Baudin et al. 2011; Mosser et al. 2012a) the physical picture is quite different for a red giant compared to the Sun and it needs a particular attention. Indeed, as shown by Figure 3 there is a clear dependence of the maxmum height of the power spectrum to the frequency $\nu_{\max }$ which is not obvious to reproduce using only our knowledge of mode amplitude in main-sequence stars. This motivated several attempts to reproduce the evolution of mode amplitudes across the HR diagram using empirical scaling relations (e.g., Kjeldsen \& Bedding 2011; Huber et al. 2011; Corsaro et al. 2013) as well as theoretical studies (Samadi et al. 2012).

To gain knowledge on mode amplitudes in red giants, the first step consisted in considering radial mode amplitudes. To do so, let us first recall that the meansquared surface velocity of a mode is given by (Samadi 2011)

$$
v^{2}(\nu, r)=\frac{\tau(\nu)}{2} \frac{P(\nu)}{\mathcal{M}(\nu, r)}
$$

where $\nu$ is the modal frequency, $\tau$ is the mode life-time (which is equal to the inverse of the mode damping rate $\eta), r$ is the radius in the atmosphere at which 
the mode velocity is measured, $P$ is the excitation rate, and $\mathcal{M}$ is the mode mass defined by

$$
\mathcal{M}=\frac{I}{\left|\xi_{r}(R)\right|^{2}}=\frac{1}{\left|\xi_{r}(R)\right|^{2}} \int_{0}^{R}\left(\xi_{r}^{2}+\Lambda \xi_{h}^{2}\right) \rho r^{2} \mathrm{~d} r
$$

where $\rho$ is the density, and $R$ is the total radius of the star.

Therefore, to reproduce the scaling relation as shown by Figure 3, one must be able to provide physically-grounded scaling relations for the mode lifetimes, mode excitation rates, and mode masses. This is a non-trivial task since the underlying physics is complex and depends on the still poorly-known upper-most layers of solar-like stars. To cope with this problem, Samadi et al. (2012) proposed to use a grid of 3D hydrodynamical simulations to determine scaling relations for both the excitation rates and mode masses at $\nu_{\max }$. For $\tau\left(\nu_{\max }\right)$, it is also possible to use the relation derived by Belkacem et al. (2012). Consequently, the amplitudes can be written such as

$$
v_{\max }=v_{\max , \odot}\left(\frac{T_{\mathrm{eff}}}{T_{\mathrm{eff}, \odot}}\right)^{-0.75}\left(\frac{\nu_{\max }}{\nu_{\max , \odot}}\right)^{-1.15}\left(\frac{\Delta \nu}{\Delta \nu_{\odot}}\right) .
$$

where $T_{\text {eff }}$ is the effective temperature, $\nu_{\max }$ the frequency of the maximum height in the oscillation power spectrum, and $\Delta \nu$ the large separation. The symbol $\odot$ denotes the solar reference values. Equation (2.3) then enable us to estimate the variation of mode amplitude with the evolution of a star when leaving the mainsequence. Indeed, as a star evolves, its surface gravity and effective temperature decrease so that both the large separation and the frequency $\nu_{\max }$ significantly decrease. It results in a significant increase of the mode amplitudes, together with a decrease of the mode linewidths, and it explains why so many red giants have been observed with solar-like oscillations.

However, most of the observations are obtained using photometric facilities and mainly with CoRoT and Kepler space-scrafts. Therefore, in order to compare predicted and measured mode amplitudes, it is necessary to convert mode-velocity amplitudes to intensity amplitudes and more precisely to luminosity perturbations $\delta L / L$ where $\delta L(t)$ is the mode Lagrangian (bolometric) luminosity perturbations. This is a non-trivial task and a consistent calculation requires to take into account the energy gain/lost by the pulsation. This can be done by using a non-adiabatic pulsation code that takes into account coupling between oscillation, radiation and turbulent convection. Due to the difficulties of consistently treating the underlying mechanisms, the use of the quasi-adiabatic relation has been proposed by Kjeldsen \& Bedding (1995) as well as Severino et al. (2008). However, those simple estimates fail to reproduce the observations and one needs to abandon the quasiadiabatic assumption which is not justified in the upper-most layers of red giants. It thus motivated Samadi et al. (2012) to use a more sophisticated approach and more precisely the MAD non-adiabatic pulsation code (Grigahcène et al. 2005). They established that the ratio between luminosity and velocity scales as the ratio 
$(L / M)^{\alpha}$ with $\alpha=0.25$ (see Samadi et al. 2012, 2015, for details). Using this result, it is quite straightforward to show that

$$
\frac{\delta L}{L} \propto T_{\mathrm{eff}}^{0.125} \nu_{\max }^{-0.75}
$$

Because we are considering red giant stars, the dependence with the effective temperature in Equation (2.4) is negligible, and we mainly have $\delta L / L \propto \nu_{\max }^{-0.75}$. When compared to the fit on the Kepler observations (see Fig. 3) as done by Mosser et al. (2012a), the agreement is quite satisfactory. Indeed, Mosser et al. (2012a) obtained $\delta L / L \propto \nu_{\max }^{-0.751}$ (see their Table 3). Therefore, one can conclude that the main physics underlying radial mode amplitudes is grasped. However, there are still some discrepancies identified by Samadi et al. (2012) to be due to both the conversion between luminosity and velocity amplitudes as well as non-adiabatic effects affecting mode driving.

\subsection{Non-radial mode amplitude for evolved stars}

Let us now consider the case of non-radial modes. The absolute amplitudes of those modes is subject to the same uncertainties as for radial modes as well as an additional difficulty related to the inner radiative damping. To focus on the latter, we will then consider the ratio between dipolar and radial modes of comparable frequencies. For sake of clarity, we will also consider that all modes are resolved, i.e. their lifetimes are smaller than the duration of the observations $\left(\tau \ll T_{\text {obs }}\right)$. In practise this is not always the case and this introduces additional subtleties but we refer the reader to Dupret et al. (2009) and Grosjean et al. (2014) for a detailed discussion in this situation.

As shown by Benomar et al. (2014), the ratio between mode heights in the power spectrum reads

$$
\frac{H_{\ell}}{H_{0}}=\left(\frac{P_{\ell}}{P_{0}}\right)\left(\frac{\mathcal{M}_{0}}{\mathcal{M}_{\ell}}\right)\left(\frac{\eta_{0}}{\eta_{\ell}}\right)^{2},
$$

where radial modes $(\ell=0)$ are denoted by the subscript 0 , and non-radial modes by the subscript $\ell$. In term of mode amplitude, it gives

$$
\frac{A_{\ell}^{2}}{A_{0}^{2}}=\left(\frac{P_{\ell}}{P_{0}}\right)\left(\frac{\mathcal{M}_{0}}{\mathcal{M}_{\ell}}\right)\left(\frac{\eta_{0}}{\eta_{\ell}}\right),
$$

where the relation between mode amplitude and mode height is $A^{2}=H \eta / 2$ (see for instance Libbrecht 1988; Baudin et al. 2005; Chaplin et al. 2005; Belkacem et al. 2006). Note that in our notation, the mode amplitude $A$ can be expression in term of both mode velocity or luminosity fluctuations as discussed in Section 2.1.

To go further, we note that at similar frequencies, the eigenfunctions of radial and non-radial modes have similar shapes in the super-adiabatic layers (i.e., near the photosphere). Therefore, the work done by the driving source on the modes is the same. This has been shown by Dupret et al. (2009) and Benomar et al. 
(2014). Obviously, for very low frequencies and high angular degrees, this is no longer verified since the horizontal component of the eigenfunction for non-radial modes become non-negligible. This leads to

$$
P_{0} \mathcal{M}_{0} \simeq P_{\ell} \mathcal{M}_{\ell} .
$$

Finally, using Equation (2.6) together with Equation (2.7), one obtains the desired relation for the mode amplitude ratio

$$
\frac{A_{\ell}^{2}}{A_{0}^{2}} \simeq\left(\frac{\mathcal{M}_{0}}{\mathcal{M}_{\ell}}\right)^{2}\left(\frac{\eta_{0}}{\eta_{\ell}}\right) .
$$

Equation (2.8) is quite enlightening because it explicitly shows that mainly two physical ingredients are susceptible to affect the amplitude of non-radial modes compared to the radial ones, namely; the mode trapping (through the inertia ratio) and an extra-contribution of mode damping. We also note that in all cases ${ }^{1}$ one has $A_{\ell} \leq A_{0}$. Indeed, if non-radial modes are efficiently trapped in the core or experience a strong damping in the core, their amplitudes will be diminished compared to the radial modes and the question of their detectability is obviously raised.

Actually, in the radiative interiors, the dominant contribution of mode damping is what we call the radiative damping since normal modes with a non-negligible amplitude lose energy radiatively. The influence of radiative damping has already been extensively investigated for other classes of pulsators (see Samadi et al. 2015, for details) and even before the unambiguous detection by CoRoT of non-radial modes in red giants, this question had been investigated from a theoretical point of view by Dziembowski et al. (2001) following the detection of variability in the red giant $\alpha$ UMa by Buzasi et al. (2000) using photometric data from the WIRE satellite. Concomitantly with the detection of non-radial stochastically excited modes in red giants by CoRoT (De Ridder et al. 2009; Dupret et al. 2009) investigated the effect of radiative damping and trapping on the amplitude of nonradial modes along with the evolution of stars with masses of $2 M_{\odot}$ and $3 M_{\odot}$. This early work was based on a full computation of mode driving as well as nonadiabatic mode damping and was extensively used to qualitatively understand the oscillation power spectrum observed by CoRoT (see the review by Mosser et al. 2016, for an account of the evolution of our understanding of redgiant spectra at the time of the CoRoT mission). This work was supplemented by Grosjean et al. (2014) who focused on low-mass red giants as mainly observed by the CoRoT and Kepler spacecrafts (see Fig. 4). The main conclusion is that radiative damping becomes dominant either for high-angular degrees or highly evolved red giant stars. In those cases, the non-radial modes can be severely affected and their amplitudes decrease so that they cannot be detected. The main physical

\footnotetext{
${ }^{1}$ Note that this inequality does not take the visibilities into account.
} 


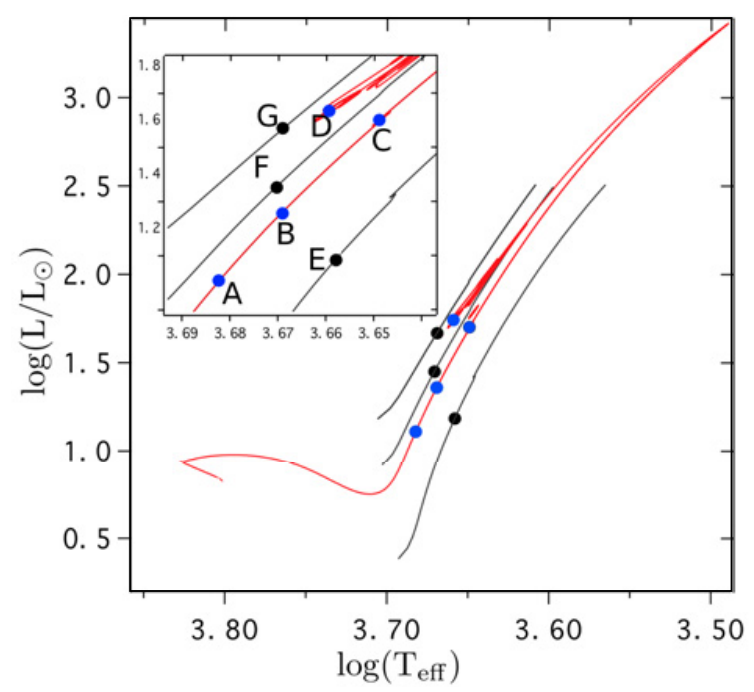

Fig. 4. Hertzprung-Russel diagram for a $1.5 M_{\odot}$ evolutionary track in red solid line and 1.7, $2 M_{\odot}$ in black. The dots are the selected models by Grosjean et al. (2014) for an in-depth study. From Grosjean et al. (2014).

ingredient is thus the ratio between the work associated with the damping in the core and in the envelope. To show it, let us remind that $\eta_{\ell}=W_{\ell} / \mathcal{M}_{\ell}$, with $W_{\ell}$ the total work integral associated to the damping (e.g. Belkacem et al. 2011), so that Equation (2.8) becomes

$$
\frac{A_{\ell}^{2}}{A_{0}^{2}} \simeq\left(\frac{\mathcal{M}_{0}}{\mathcal{M}_{\ell}}\right)\left(\frac{W_{0}}{W_{\ell}}\right) .
$$

To go further, it is useful to write $W_{\ell}=W_{\text {env }}+W_{\text {core }}$, where $W_{\text {env }}$ and $W_{\text {core }}$ are the work integrals associated to the enveloppe and the core, respectively. Moreover, using the same argument as for the driving (see Eq. (2.7)) one can assume $W_{\text {env }}=W_{0}$ so that

$$
\frac{A_{\ell}^{2}}{A_{0}^{2}} \simeq\left(\frac{\mathcal{M}_{0}}{\mathcal{M}_{\ell}}\right)\left(1+\frac{W_{\text {core }}}{W_{0}}\right)^{-1} .
$$

The evolution of the ratio $W_{\text {core }} / W_{0}$ is illustrated by Figure 5 and its influence on the shape of the oscillation power spectra is shown in Figure 6. Guided by the above-mentioned arguments and using complete computations of mode driving and damping, Grosjean et al. (2014) were able to theoretically predict the limit of mixed mode detectability commensurable with the observations. The authors also demonstrated that the structure of oscillation spectra is identical for a given ratio of the number of mixed modes between two consecutive $p$ modes $\left(n_{g} / n_{p}\right)$ as depicted by Figure 7 .

Finally, it is worthwhile to note that the amplitude ratio can be easily estimated using asymptotic developments. From Equation (2.10), there are two distinct 

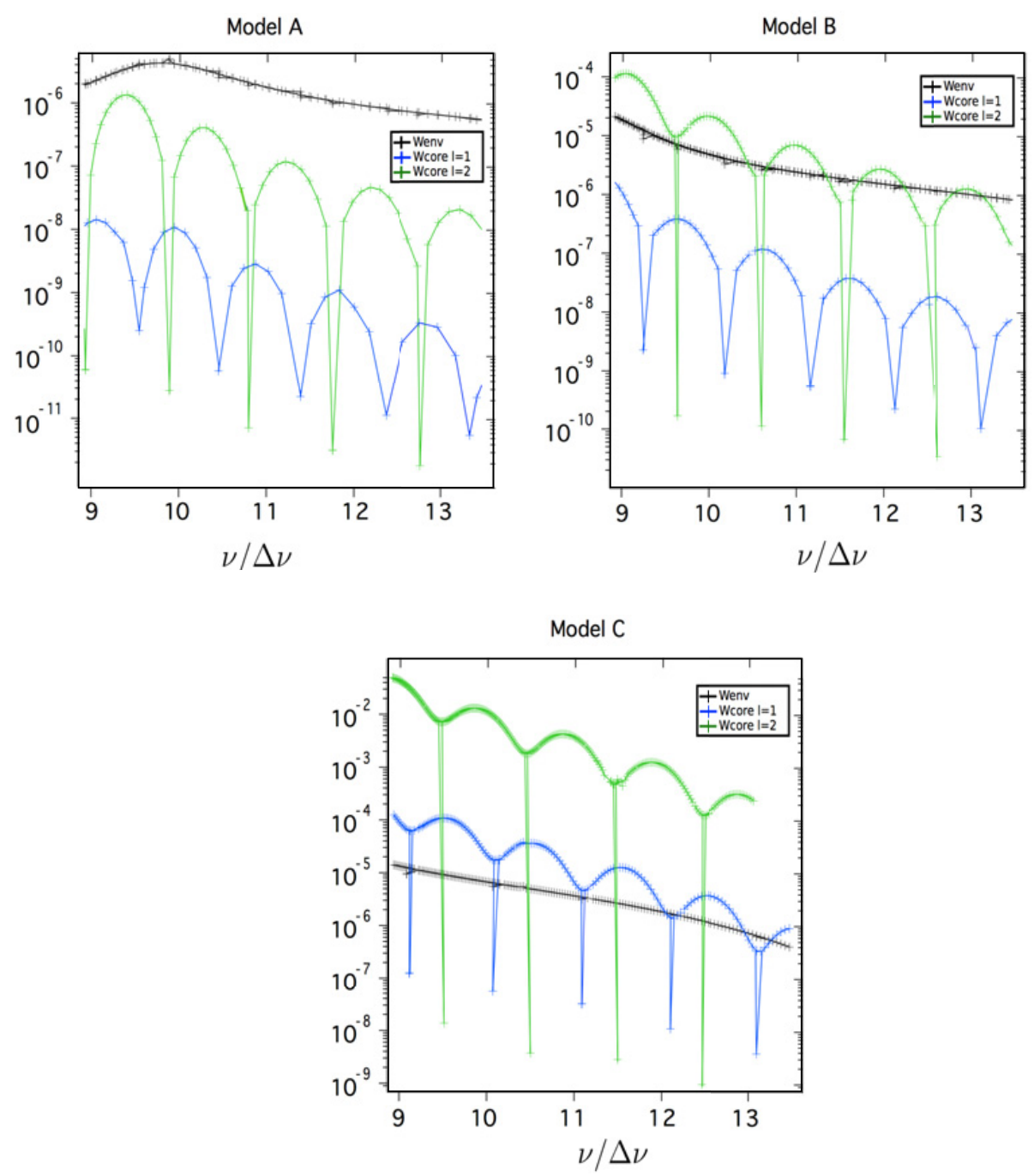

Fig. 5. Core and envelope contributions to the work integral, normalised by $G M^{2} / R$, for the three models A,B,C (from top to bottom panels, respectively) of the $1.5 \mathrm{M}$ evolutionary tracks as shown by Figure 4. From Grosjean et al. (2014).

cases, namely;

- if the work associated with the radiative damping in the core is negligible compared to the work associated with the damping in the envelope, the mode amplitude ratio Equation (2.10) reads (see also Belkacem et al. 2015a)

$$
\frac{A_{\ell}^{2}}{A_{0}^{2}} \simeq\left(\frac{\mathcal{M}_{0}}{\mathcal{M}_{\ell}}\right)=1-\zeta,
$$

where we have introduced the now commonly used $\zeta$ function (e.g. Goupil et al. 2013; Mosser et al. 2015) that represents the ratio between the mode 

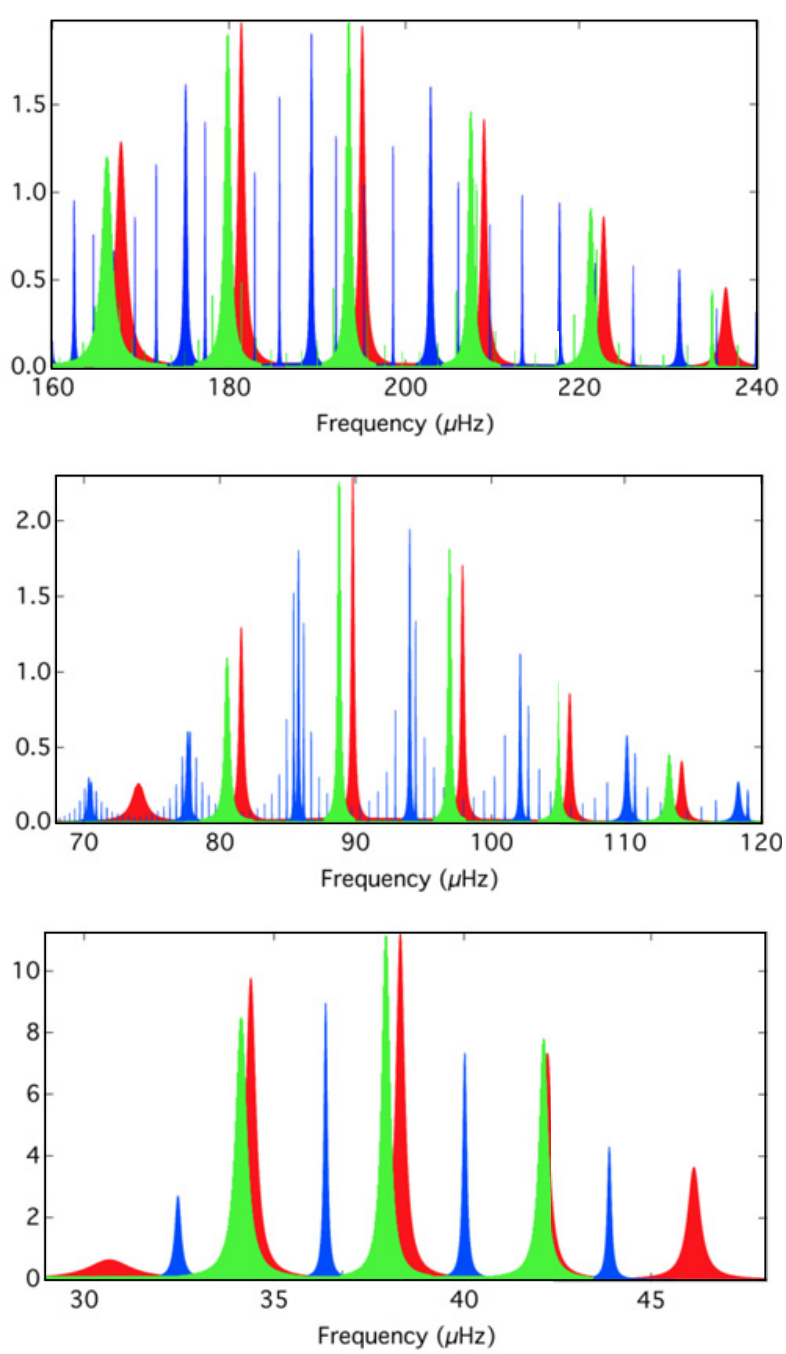

Fig. 6. Power spectra of models A, B, and C (see Fig. 4). The heights are given in $m / s)^{2} / \mu \mathrm{Hz}$. From Grosjean et al. (2014).

inertia in the core and the total mode inertia. As we will show, in the following section, this function can be easily determined using asymptotic developments. Equation (2.11) holds for the low angular degrees in subgiants and early red giants.

- if $W_{\text {core }}$ cannot be neglected compared to $W_{0}$ one can also estimate the amplitude ratio using asymptotic developments by writting

$$
\frac{A_{\ell}^{2}}{A_{0}^{2}} \simeq \frac{1-\zeta}{1+\frac{\eta_{\text {core }}}{\eta_{0}} \zeta},
$$




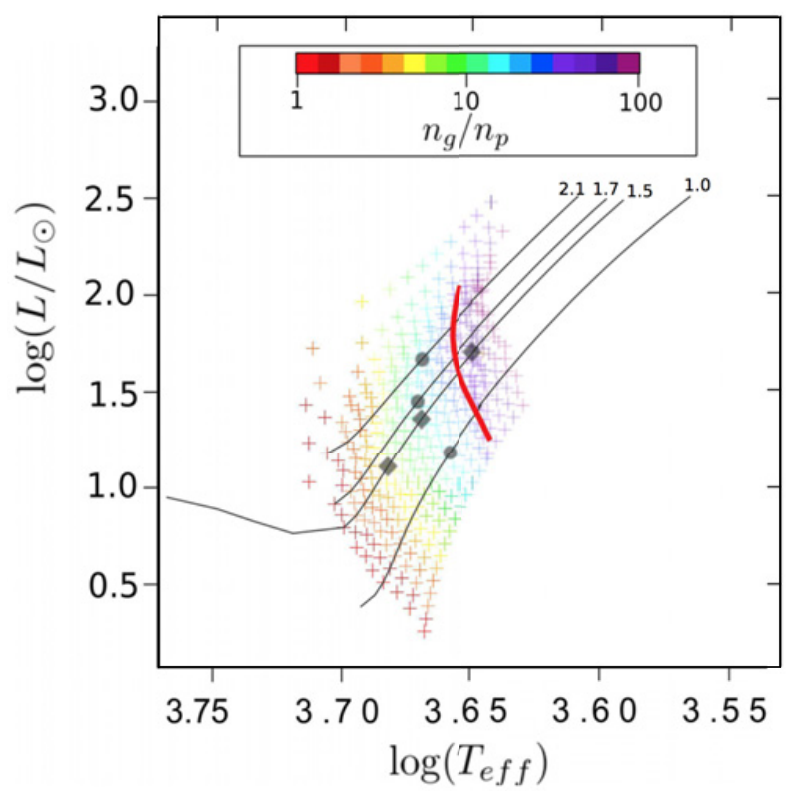

Fig. 7. The same as in Figure 4 except that the color scale indicates models with the same number of mixed modes by large separation. The red line represents the detectability limit found for the dipole modes by Grosjean et al. (2014) (assuming that all modes are fully resolved). From Grosjean et al. (2014).

where $\eta_{0}$ can be easily determined using observations or scaling relation as for instance given by Belkacem et al. (2012), and $\eta_{\text {core }}$ is the radiative damping which can be written as (e.g. Dziembowski et al. 2001; Godart et al. 2009)

$$
\eta\left(\ell_{\max }, \nu\right)=\frac{\left[\ell_{\max }\left(\ell_{\max }+1\right)\right]^{3 / 2}}{8 \pi \sigma_{R}^{3} \int_{0}^{R_{c}} k_{r} \mathrm{~d} r} \int_{0}^{R_{c}} \frac{\nabla_{\mathrm{ad}}-\nabla}{\nabla} \frac{\nabla_{\mathrm{ad}} N g L}{P r^{5}} \mathrm{~d} r .
$$

where $k_{r}^{2} \simeq \frac{\ell(\ell+1)}{r^{2}}\left(\frac{N^{2}}{\sigma_{R}^{2}}\right), N$ is the buoyancy frequency, $P$ is the pressure, $L$ is the luminosity, $R_{c}$ is the base of the convective region, $\sigma_{R}=2 \pi \nu, \nabla$ is the temperature gradient and $\nabla_{\text {ad }}$ its adiabatic counter-part.

Finally, one can conclude that the main physics underlying the oscillation power spectra of evolved stars among the HR diagram is now grasped. It was a crucial and even mandatory step before using the mixed modes for inferring the internal structure of red giants. However, even if the structure and evolution of the spectra is understood, one still must be able to properly know what king of information bear the oscillation frequencies and this is not a trivial task as shown in the following section. 


\section{Mixed mode frequencies: Disentangling mode trapping, glitches, and rotational splittings}

Once detected, mixed modes are not directly usable to infer the internal structure of evolved stars. Indeed, a number of physical mechanisms influence their frequencies. The main challenge is thus to disentangle all the contributions. This is a non-trivial task and quite a number of studies focused on that objective (see the review by Hekker \& Christensen-Dalsgaard 2016) but it is safe to realize that all those works rely directly or implicitly on asymptotic expansions. They allow us to link the frequencies with structural properties of stars in idealized situations. Asymptotic developments are therefore unique and essential tools to gain knowledge on the structure of oscillation spectra.

\subsection{Asymptotic expansion for mixed modes}

Pure gravity mode frequencies are equally spaced in periods. It is the counterpart of the large separation for pure acoustic modes. Indeed, gravity mode periods follow the relation (Tassoul 1980)

$$
\Pi=\Delta \Pi\left(n+\epsilon_{g}\right),
$$

where $\epsilon_{g}$ is a phase shift, $n$ is the radial order, and $\Delta \Pi$ is the period spacing given by

$$
\Delta \Pi=\frac{2 \pi^{2}}{\sqrt{\ell(\ell+1)}}\left(\int_{r_{1}}^{r_{2}} N \frac{\mathrm{d} r}{r}\right)^{-1},
$$

where $r_{1}$ and $r_{2}$ are the radius of the inner and outer turning points of the $g$-mode cavity. It is worth to note that the integral in Equation (3.2) is related to the evolutionary state of the star since it provides information on the inner-most layers of evolved stars. For instance, it has been possible to unambiguously distinguish between low-mass stars on the ascending red-giant branch (RGB) and on the central helium burning phase (clump stars) by using the period spacings while it was impossible to conclude only with surface properties such as effective temperature and surface gravities (Bedding et al. 2011; Mosser et al. 2011). For both RGB and clump stars it is possible to go a step further since the period spacing is very sensitive to the internal structure of the core and thus to the evolutionary stage.

However, if one uses directly the period spacing between two consecutive mixed modes, $\Delta P$, as in Bedding et al. (2011) and Mosser et al. (2011), the diagnostic remains limited. Actually, the period spacing between two consecutive observed mixed modes does not follow exactly the asymptotic relation as given by Equation (3.2). This is related to the mixed nature of the modes. The observed modes are not pure $g$-modes because they are affected by their acoustic nature in the envelope that induces a departure from Equation (3.2). This is illustrated by Figure 8.

To cope with this issue, Mosser et al. (2012a) proposed an asymptotic relation adapted for mixed modes, essentially a matching between asymptotic solution in 


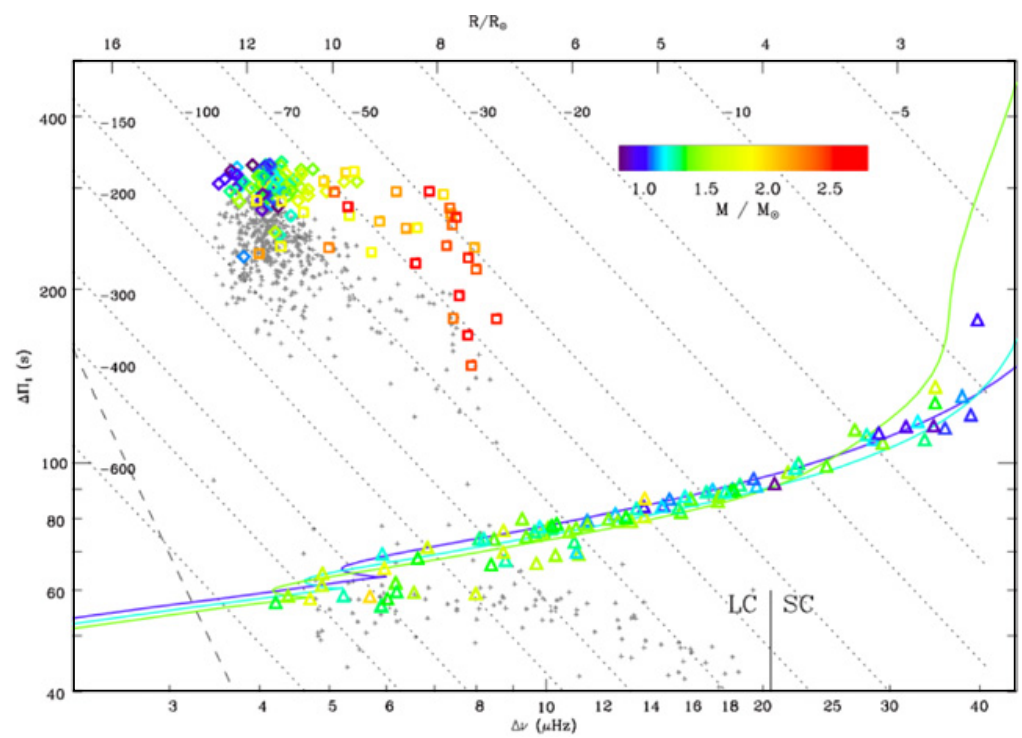

Fig. 8. Gravity-mode period spacing $\Delta \Pi$ as a function of the pressure-mode large frequency spacing $\Delta \nu$. RGB stars are indicated by triangles; clump stars by diamonds; secondary clump stars by squares. The seismic mass is given by the color code. Small gray crosses indicate the bumped periods $\Delta P$. The solid colored lines correspond to a grid of stellar models with masses of $1,1.2$ and $1.4 M_{\odot}$, from the ZAMS to the tip of the RGB. From Mosser et al. (2012a).

the acoustic and buoyancy cavities (this is based on the original work by Shibahashi 1979b). It allows us to express the mixed mode frequencies as a function of $p$-mode frequencies, i.e.

$$
\nu=\nu_{n_{p}}+\frac{\Delta \nu}{\pi} \arctan \left[q \tan \pi\left(\frac{1}{\Delta \Pi \nu}-\epsilon_{g}\right)\right],
$$

where $\nu$ is the mixed mode frequency, $\nu_{n_{p}}$ the frequency of pure $p$ modes, and $q$ a coupling factor. Therefore, using Equation (3.3) with the measured $\nu$ yields a determination of the asymptotic period spacing $(\Delta \Pi)$ through a simple fitting. This is a crucial step for comparing the observations and the modelling as well as for determining the evolutionary stage (Mosser et al. 2014; Vrard et al. 2016). As illustrated by Figure 8, the observed asymptotic period spacing can be easily compared to the period spacing computed by the models using Equation (3.2).

It is possible to go a step further and to relate directly $\Delta P$ and $\Delta \Pi$ by considering that it mainly depends on the ratio between the inertia contribution in the $g$-mode cavity and the total inertia of a mixed mode. More precisely, one has (Christensen-Dalsgaard 2012; Mosser et al. 2015)

$$
\Delta P=\Delta \Pi \zeta
$$


where an asymptotic expression of $\zeta$ can be obtained as demonstrated by Goupil et al. (2013) and generalized by Deheuvels et al. (2015) and Mosser et al. (2015), i.e.

$$
\zeta(\nu)=\left[1+\frac{1}{q} \frac{\nu^{2} \Delta \Pi}{\Delta \nu\left(\nu_{p}\right)} \frac{\cos ^{2} \frac{\pi}{\Delta \Pi}\left(\frac{1}{\nu}-\frac{1}{\nu_{g}}\right)}{\cos ^{2} \pi \frac{\nu-\nu_{p}}{\Delta \nu\left(\nu_{p}\right)}},\right]^{-1} .
$$

where $\nu_{p}$ and $\nu_{g}$ are the frequencies of pure $p$ and $g$ modes, respectively.

This ratio is fundamental for understanding mixed mode spectra because, at leading order, it controls almost all the physical parameters such as mode amplitudes (see Sect. 2), period spacing, but also rotational splittings as we will discuss in Section 3.2. Therefore, for inferring precisely the internal structure of red giants, it is essential to remove this dominant dependency. It motivated the work by Mosser et al. (2015). The authors proposed an elegant way for extracting higher-order effects from the mixed mode frequencies by introducing a change of variable, named to as stretched periods. Assuming that the frequencies and the periods are continuous functions, one can write

$$
\mathrm{d} \tau=\frac{1}{\zeta} \frac{\mathrm{d} \nu}{\nu^{2}}
$$

where $\tau$ is the stretched period and $\zeta$ is given by Equation (3.5). Figure 9 displays an example for a star observed by Kepler in which the rotation is not detected.

\subsection{Measurements of core rotation and evidence of spin-down}

When rotation is non-negligible, non-antisymmetric modes (i.e. with $m \neq 0$, where $m$ is the azimuthal) can be detected albeit it depends on the inclination of the star. Therefore, the frequency difference between the modes with $m=0$ and $m \neq 0$ can be measured. These are the rotational splittings, $\delta \nu$ (see Goupil 2011 for a review on the influence of rotation on mode frequencies). In red giants, the frequency dependence of the splittings with the frequency is intimately intertwined with the frequency dependence of $\Delta P$. To disentangle between the effects of the trapping (through $\zeta$ ) and the rotation, the stretched periods are again very useful. If one defines $\delta \tau$ as the difference between the stretched periods of two modes of consecutive radial orders and the same azimuthal order, one has (Mosser et al. 2015)

$$
\delta \tau=\Delta \Pi+\frac{2 m \delta \nu}{\nu} \Delta P=\Delta \Pi\left(1+\frac{2 m \delta \nu}{\nu} \zeta\right) .
$$

Note that Equation (3.7) has been derived assuming the rotation of the envelope can be neglected. This is quite a safe assumption for RGB stars and particularly for $g$-dominated modes. Equation (3.7) immediately shows that an échelle diagram using the stretched period modulo $\Delta \Pi$ immediately exhibits the rotational splittings as illustrated by Figure 10. 

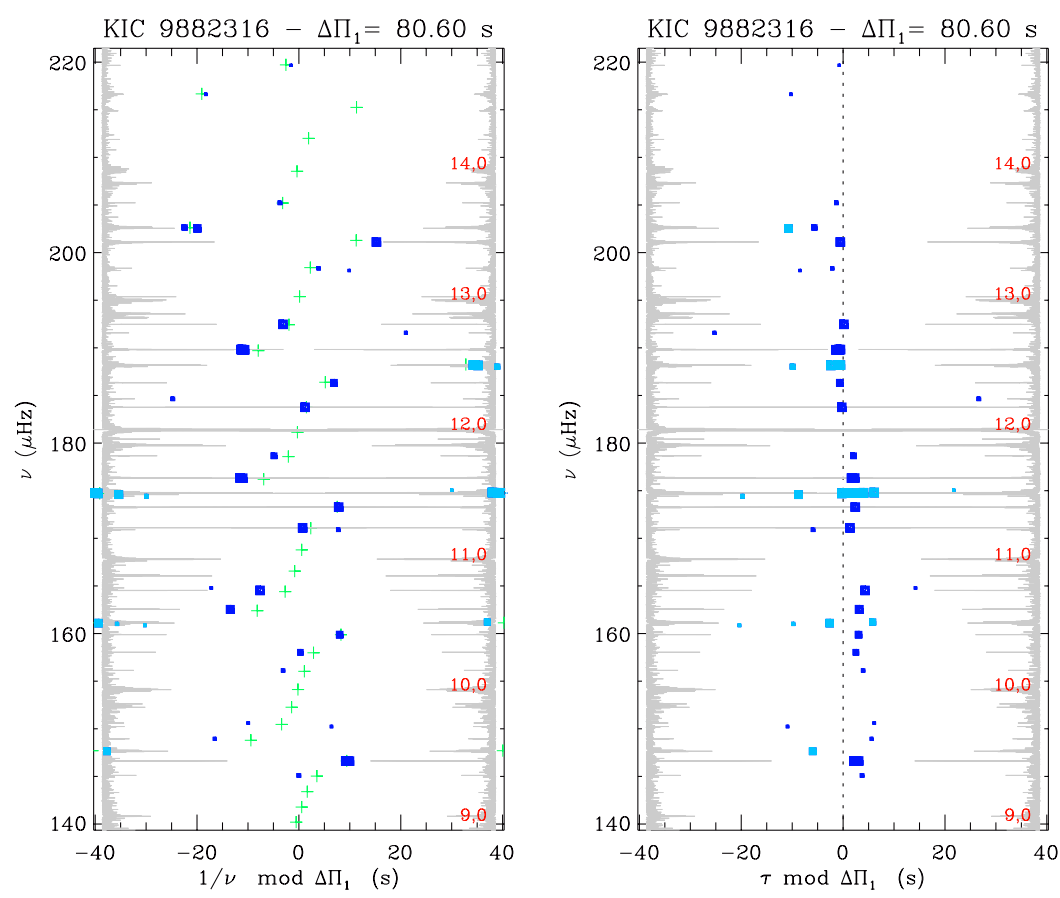

Fig. 9. Échelle diagrams of the RGB star KIC 9882316. The left panel shows the classical period chelle diagram, where the abscissa is the period modulo the period spacing. The most prominent mixed modes are marked with blue filled squares (in light blue for peaks in the vicinity of $p$-dominated modes). The right panel displays the stretched period chelle diagram, where the abscissa is the stretched period modulo the period spacing. Pressure-dominated mixed modes are coded in light blue. From Mosser et al. (2015).

One must now relate the rotational splittings $(\delta \nu)$ and the mean rotation of the core. As above-mentioned and demonstrated by Goupil et al. (2013) in the case of linear splitting (see Ouazzani et al. 2013 when rotation can no longer be treated as a perturbation), the ratio $\zeta$ is still an important factor and one has for $g$-dominated modes

$$
\delta \nu \simeq \frac{1}{2}\left\langle\frac{\Omega}{2 \pi}\right\rangle_{\text {core }} \zeta
$$

where $\Omega$ is the rotational angular frequency. Before going further, it is fundamental to stress out that the symbol \langle\rangle$_{\text {core }}$ denotes the seismic average and must not be directly compared to the core rotation rate inferred in models as unfortunately done in several works. Indeed, one has

$$
\left\langle\frac{\Omega}{2 \pi}\right\rangle_{\text {core }}=\frac{1}{2 \pi}\left(\int_{0}^{x_{\text {core }}} \Omega(x) K(x) \mathrm{d} x\right)\left(\int_{0}^{x_{\text {core }}} K(x) \mathrm{d} x\right)^{-1},
$$




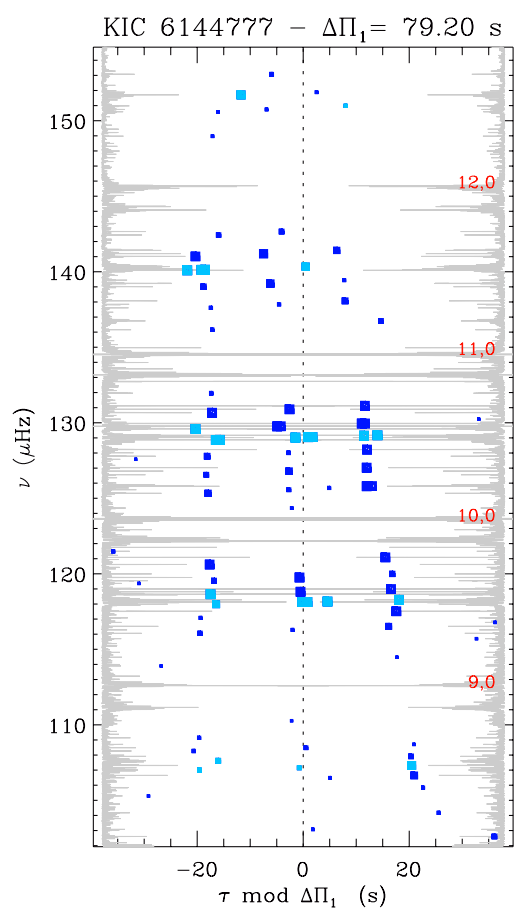

Fig. 10. The same as for Figure 9 but for the star KIC 6144777. The three main ridges are due to the dipolar modes and more precisely to the $m=-1,0,1$ modes. From Mosser et al. (2015).

with $x_{\text {core }}$ the normalized radius of the upper turning point of the $g$ modes which can be approximated by the normalized radius of the radiative core, and

$$
K(x)=\frac{1}{I}\left(\xi_{r}^{2}+(\Lambda-1) \xi_{h}^{2}-2 \xi_{r} \xi_{h}\right) \rho x^{2},
$$

where $I$ the mode inertia.

Using first-order asymptotic developments as well as numerical calculation, Goupil et al. (2013) have shown that this seismic average can be approximated by

$$
\left\langle\frac{\Omega}{2 \pi}\right\rangle_{\text {core }} \simeq \frac{1}{\tau_{g}} \int_{0}^{x_{\text {core }}} \frac{\Omega(x)}{2 \pi} \frac{N}{x} \mathrm{~d} x,
$$

where $N$ is the buoyancy frequency and $\tau_{g}$ is the time spent in the $g$-mode cavity by a mode defined by

$$
\tau_{g}=\int_{0}^{x_{\text {core }}} N \frac{\mathrm{d} x}{x} .
$$

Therefore, one must be cautious when comparing seismic core rotation of evolved stars with rotation rates derived from evolutionary models because, as shown by 


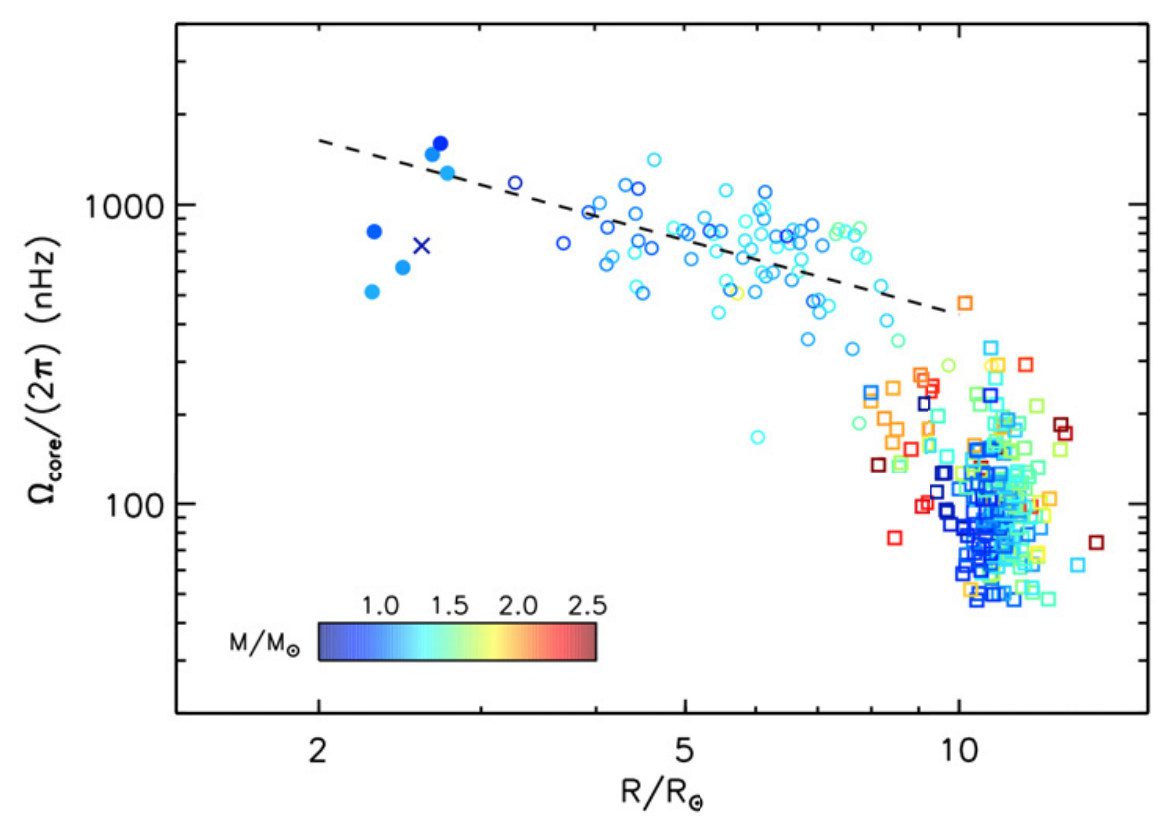

Fig. 11. Rotation rate of the core as a function of the stellar radius. The open dots and open squares correspond to the RGB and clump stars, respectively, investigated by Mosser et al. (2012b). The filled symbols indicate the subgiant stars studied by Deheuvels et al. (2012, 2014). Figure from Deheuvels et al. (2015).

Equation (3.11), the seismic average crucially depends on the rotation profile and how it compares with the buoyancy frequency profile. Moreover, it allows ones to see that the seismic average probes the rotation rate in the inner most layers where $N$ is important. Indeed, it is of no help for the rotation rate in-between the hydrogen burning shell and the base of the upper convective zone.

Such a methodology has been used by Mosser et al. (2012b) to infer core rotation of hundreds of RGB and clump stars. For less-evolved stars, namely subgiant stars, Deheuvels et al. $(2012,2014)$ used a different methodology by using inversion techniques. It was made possible mainly because the reciprocal influence of the $p$ and $g$ cavities are less severe so that the information is less degenerated than for more evolved RGB stars. The overall result is well illustrated by Figure 11. Since the total radius is a proxy of the evolutionary stage, one can draw several conclusions, namely

- For subgiant stars, even if the number of stars remains limited, it seems that the radiative core spins-up as expected by considering the local conservation of angular momentum (hereafter AM). Indeed, when a star evolves off-mainsequence, its core contracts. However, anticipating on the following section, the spin-up is not high enough to be compatible with the local conservation 
of AM. It means that angular momentum transport is needed to explain the observation.

- For red giant stars, the situation is even more critical since their core spindown along with their evolution. It is the signature of a powerful physical mechanism able to redistribute AM from the core to the envelope.

- The edge between subgiants and redgiants clearly raises a number of questions. Does the slope change results from a different physical mechanism of AM transport at work and does it simply comes from a difference in the efficiency of a single physical process? The lack of observational data clearly amplifies the questioning but we need to rely on modelling before space-borne missions such as PLATO (Rauer et al. 2014) would be able to conclude.

Finally, we can draw a relatively simple conclusion. One or several physical processes must be invoked to explained the efficient angular momentum transport from the core to the envelope of evolved stars.

\section{The aftermath: The quest for angular momentum transport}

With the measurement of the core rotation of evolved stars it was soon realized that current models including angular momentum redistribution processes are unable to explain the spin-down of red giant stars. Eggenberger et al. (2012) investigated the particular case of the star KIC8366239 observed by Kepler and tried to reproduce the rotational splittings as measured by Beck et al. (2012). Within the approximation of shellular rotation (Zahn 1992), the authors included the angular momentum advection by meridional circulation as well as diffusion of AM by shear mixing and showed that using classical values for turbulent viscosities is not sufficient. This was independently confirmed by Marques et al. (2013) (see also Ceillier et al. 2013) who showed that even by accounting for the large uncertainties related to the horizontal viscosities as well as to the surface braking law meridional circulation and shear instabilities are unable to explain the low rotation rates of subgiants and red giants. Note, however, that the issue is not completely settled because the the horizontal diffusion coefficient is still subject to discussion (see L. Amard in this Volume). Eggenberger et al. (2012) also proposed to constrain the unknown additional mechanism by fitting the turbulent viscosity. This has been be generalized by Eggenberger et al. (2017) who showed that the efficiency of the missing mechanism increases with the stellar mass. We note, however, that such an approach, while putting stringent constraint on the AM transport, assumes that the additional mechanism is a diffusion process.

Meridional circulation and shear instabilities being helpless for explaining the observations, the potential contribution of the magnetic field was soon to be addressed. Cantiello et al. (2014) investigated the effect of a magnetic field generated through the Tayler-Spruit dynamo (see the seminal papers by Spruit 1999, 2002) and reached almost the same negative conclusion. An alternative mechanism, 
involving magnetic field, was proposed by Rüdiger et al. (2015). The authors investigated the effect of the magneto-rotational instabilities of a toroidal magnetic field (AMRI). They suggest that it could explain the angular momentum redistribution in subgiants and early red giants (see also Maeder \& Meynet 2014). Even if their modelling is idealized, for instance assuming chemically homogeneous fluid, the transport of AM by an azimuthal magneto-rotational instability as proposed by Rüdiger et al. (2015) constitutes a promising candidate to further investigate. Interestingly enough, Spada et al. (2016) fitted to the observations a power law (of the ratio between the rotation frequency of the radiative and convective layers) for the AM diffusion coefficient and showed that the power is compatible with the AMRI.

A second class of non-standard physical process can help in solving the problem of the rotation of evolved stars, namely; waves. This includes both progressive (hereafter mentioned as waves) and standing waves (hereafter mentioned as modes). The former, and mainly internal gravity waves (IGW) can transport angular momentum (e.g., Press 1981) and it has been demonstrated that they could explain the nearly flat rotation profile in the inner radiative zone of the Sun (Charbonnel \& Talon 2005). For evolved stars, Fuller et al. (2014) have found that internal gravity waves are likely to couple the convective region and the upper radiative region, but not the innermost layers, i.e. the core of redgiants. More precisely, they demonstrated that IGW could not reach the core of RGB stars because they are highly damped near hydrogen burning shell (i.e. near the peak of the buoyancy frequency). We note however, that it also means a strong local extraction of AM which can potentially have non-negligible influence on other processes such as meridional circulation. This interaction as well as many others remain a weak point in current modelling of angular momentum transport and would deserve attention in the future. In contrast, for subgiants and early redgiants, the recent work by Pinçon et al. (2016b) provides new insight on the role of IGW. Based on the formalism developed by Pinçon et al. (2016a) to model excitation of IGW by convective plumes, the authors suggest that IGW could explain the observed differential rotation of subgiants and early red giants as observed by Deheuvels et al. (2014).

However, for more evolved red giants, IGW are helpless but mixed modes can be decisive. Indeed, mixed modes allowed us to unveil the internal rotation of evolved stars but they can also play an active role. Actually, redistribution of angular momentum by normal modes has been considered for a long time with the pioneer work by Ando (1983) who addressed the question of the interaction of wave and rotation through wave momentum stresses in the mean angular momentum equation, and many works followed in the context of massive stars (e.g., Ando 1986; Lee \& Saio 1993; Lee 2007; Townsend \& MacDonald 2008; Townsend 2014; Lee et al. 2014). To estimate the influence of mixed modes in low-mass evolved stars, Belkacem et al. (2015a, 2015b) revisited the formalism to account properly for the influence of both the wave momentum and heat fluxes on the mean flow. Then, using realistic estimates of mode amplitudes, they were able to show that mixed modes are able to efficiently slow-down RGB stars but not subgiants. Thus, 
one can conclude that the combination of IGW for sugiants and mixed modes for RGB stars is a competitive candidate to account for the observations. No doubts this will be further investigated in the near future to reach a definitive conclusion.

\section{Concluding remarks}

Finally, since the unambiguous detection of mixed modes in red giants by CoRoT (De Ridder et al. 2009), huge efforts have been made to unveil the interior structure and rotation of these stars. They were highly successful and we can bet that we are only at the beginning of the story. The analysis of existing observational data are still ongoing and the forthcoming grounded-based, such as SONG facility (Grundahl et al. 2006), and space-borne missions, such as TESS (Ricker et al. 2015) and PLATO (Rauer et al. 2014), will certainly be decisive to grasp to physics of angular momentum transport in low-mass stars. Jean-Paul Zahn has always been at the forefront in this topic and no doubt much of the recent advances would have not been made possible without his precursor contributions.

K. B. is grateful to the organizers for their invitation. The author also thanks B. Mosser and R. Samadi for reading the manuscript and for providing useful remarks.

\section{References}

Ando, H., 1983, PASJ, 35, 343

Ando, H., 1986, A\&A, 163, 97

Appourchaux, T., Belkacem, K., Broomhall, A.-M., et al., 2010, A\&A Rev., 18, 197

Baudin, F., Barban, C., Belkacem, K., et al., 2011, A\&A, 529, A84

Baudin, F., Samadi, R., Goupil, M.-J., et al., 2005, A\&A, 433, 349

Beck, P.G., Montalban, J., Kallinger, T., et al., 2012, Nature, 481, 55

Bedding, T.R., Mosser, B., Huber, D., et al., 2011, Nature, 471, 608

Belkacem, K., Dupret, M.A., Baudin, F., et al., 2012, A\&A, 540, L7

Belkacem, K., Goupil, M.J., Dupret, M.A., et al., 2011, A\&A, 530, A142

Belkacem, K., Marques, J.P., Goupil, M.J., et al., 2015a, A\&A, 579, A31

Belkacem, K., Marques, J.P., Goupil, M.J., et al., 2015b, A\&A, 579, A30

Belkacem, K., Samadi, R., Goupil, M.J., et al., 2009, A\&A, 494, 191

Belkacem, K., Samadi, R., Goupil, M.J., Kupka, F., \& Baudin, F., 2006, A\&A, 460, 183

Benomar, O., Belkacem, K., Bedding, T.R., et al., 2014, ApJ, 781, L29

Buzasi, D., Catanzarite, J., Laher, R., et al., 2000, ApJ, 532, L133

Cantiello, M., Mankovich, C., Bildsten, L., Christensen-Dalsgaard, J., \& Paxton, B., 2014, ApJ, 788, 93

Ceillier, T., Eggenberger, P., García, R.A., \& Mathis, S., 2013, A\&A, 555, A54

Chaplin, W.J., Houdek, G., Elsworth, Y., et al., 2005, MNRAS, 360, 859

Chaplin, W.J., \& Miglio, A., 2013, ARA\&A, 51, 353

Charbonnel, C., \& Talon, S., 2005, Science, 309, 2189 
Christensen-Dalsgaard, J., 2004, Sol. Phys., 220, 137

Christensen-Dalsgaard, J., 2012, in Astronomical Society of the Pacific Conference Series, Vol. 462, Progress in Solar/Stellar Physics with Helio- and Asteroseismology, ed. H. Shibahashi, M. Takata, \& A.E. Lynas-Gray, 503

Christensen-Dalsgaard, J., \& Frandsen, S., 1983, Solar Phys., 82, 469

Corsaro, E., Fröhlich, H.-E., Bonanno, A., et al., 2013, MNRAS, 430, 2313

De Ridder, J., Barban, C., Baudin, F., et al., 2009, Nature, 459, 398

Deheuvels, S., Ballot, J., Beck, P.G., et al., 2015, A\&A, 580, A96

Deheuvels, S., Doğan, G., Goupil, M.J., et al., 2014, A\&A, 564, A27

Deheuvels, S., García, R.A., Chaplin, W.J., et al., 2012, ApJ, 756, 19

Dupret, M.-A., Belkacem, K., Samadi, R., et al., 2009, A\&A, 506, 57

Dziembowski, W.A., 1971, Acta. Astron., 21, 289

Dziembowski, W.A., Gough, D.O., Houdek, G., \& Sienkiewicz, R., 2001, MNRAS, 328, 601

Eggenberger, P., Lagarde, N., Miglio, A., et al., 2017, A\&A, 599, A18

Eggenberger, P., Montalbán, J., \& Miglio, A., 2012, A\&A, 544, L4

Fuller, J., Lecoanet, D., Cantiello, M., \& Brown, B., 2014, ApJ, 796, 17

Godart, M., Noels, A., Dupret, M.-A., \& Lebreton, Y., 2009, MNRAS, 396, 1833

Goupil, M.-J., 2011, ArXiv e-prints

Goupil, M.J., Mosser, B., Marques, J.P., et al., 2013, A\&A, 549, A75

Grigahcène, A., Dupret, M.-A., Gabriel, M., Garrido, R., \& Scuflaire, R., 2005, A\&A, 434, 1055

Grosjean, M., Dupret, M.-A., Belkacem, K., et al., 2014, ArXiv e-prints

Grotsch-Noels, A. \& Deheuvels, S., 2016, IV.1 Insights on the internal structure of stars as provided by seismology, ed. CoRot Team, 181

Grundahl, F., Kjeldsen, H., Frandsen, S., et al., 2006, Mem. Soc. Astron. Italiana, 77, 458

Hekker, S., \& Christensen-Dalsgaard, J., 2016, ArXiv e-prints

Houdek, G., Balmforth, N.J., Christensen-Dalsgaard, J., \& Gough, D.O., 1999, A\&A, 351,582

Huber, D., Bedding, T.R., Stello, D., et al., 2011, ApJ, 743, 143

Kjeldsen, H., \& Bedding, T.R., 1995, A\&A, 293, 87

Kjeldsen, H. \& Bedding, T.R., 2011, A\&A, 529, L8

Lee, U., 2007, in Astronomical Society of the Pacific Conference Series, Vol. 361, Active OB-Stars: Laboratories for Stellare and Circumstellar Physics, ed. A.T. Okazaki, S.P. Owocki, \& S. Stefl, 45

Lee, U., Neiner, C., \& Mathis, S., 2014, MNRAS, 443, 1515

Lee, U. \& Saio, H., 1993, MNRAS, 261, 415

Libbrecht, K.G., 1988, ApJ, 334, 510

Maeder, A., \& Meynet, G., 2014, ApJ, 793, 123

Marques, J.P., Goupil, M.J., Lebreton, Y., et al., 2013, A\&A, 549, A74

Mosser, B., Barban, C., Montalbán, J., et al., 2011, A\&A, 532, A86

Mosser, B., Belkacem, K., Pinçon, C., et al., 2017, A\&A, 598, A62 
Mosser, B., Benomar, O., Belkacem, K., et al., 2014, A\&A, 572, L5

Mosser, B., Elsworth, Y., Hekker, S., et al., 2012a, A\&A, 537, A30

Mosser, B., Goupil, M.J., Belkacem, K., et al., 2012b, A\&A, 548, A10

Mosser, B., \& Miglio, A., 2016, IV.2 Pulsating red giant stars, ed. CoRot Team, 197

Mosser, B., Vrard, M., Belkacem, K., Deheuvels, S., \& Goupil, M.J., 2015, A\&A, 584, A50

Ouazzani, R.-M., Goupil, M.J., Dupret, M.-A., \& Marques, J.P., 2013, A\&A, 554, A80

Pinçon, C., Belkacem, K., \& Goupil, M.J., 2016a, A\&A, 588, A122

Pinçon, C., Belkacem, K., \& Goupil, M.J. 2016b, in SF2A-2016: Proceedings of the Annual meeting of the French Society of Astronomy and Astrophysics, ed. C. Reylé, J. Richard, L. Cambrésy, M. Deleuil, E. Pécontal, L. Tresse, \& I. Vauglin, 179-183

Press, W.H., 1981, ApJ, 245, 286

Rauer, H., Catala, C., Aerts, C., et al., 2014, Exper. Astron., 38, 249

Ricker, G.R., Winn, J.N., Vanderspek, R., et al., 2015, J. Astron. Telesc., Instrum. Syst., 1, 014003

Rüdiger, G., Gellert, M., Spada, F., \& Tereshin, I., 2015, A\&A, 573, A80

Samadi, R., 2011, in Lecture Notes in Physics, Berlin Springer Verlag, Vol. 832, Lecture Notes in Physics, Berlin Springer Verlag, ed. J.-P. Rozelot \& C. Neiner, 305

Samadi, R., Belkacem, K., Dupret, M.-A., et al., 2012, A\&A, 543, A120

Samadi, R., Belkacem, K., \& Sonoi, T., 2015, in EAS Publications Series, Vol. 73, EAS Publications Series, 111-191

Samadi, R., Georgobiani, D., Trampedach, R., et al., 2007, A\&A, 463, 297

Scuflaire, R., 1974, A\&A, 36, 107

Severino, G., Straus, T., \& Steffen, M., 2008, Sol. Phys., 251, 549

Shibahashi, H., 1979a, PASJ, 31, 87

Shibahashi, H., 1979b, PASJ, 31, 87

Spada, F., Gellert, M., Arlt, R., \& Deheuvels, S., 2016, A\&A, 589, A23

Spruit, H.C., 1999, A\&A, 349, 189

Spruit, H.C., 2002, A\&A, 381, 923

Tassoul, M., 1980, ApJS, 43, 469

Townsend, R., 2014, in IAU Symposium, Vol. 301, IAU Symposium, ed. J.A. Guzik, W.J. Chaplin, G. Handler, \& A. Pigulski, 153-160

Townsend, R. \& MacDonald, J., 2008, in IAU Symposium, Vol. 250, IAU Symposium, ed. F. Bresolin, P.A. Crowther, \& J. Puls, 161-166

Vrard, M., Mosser, B., \& Samadi, R., 2016, A\&A, 588, A87

Zahn, J.-P., 1992, A\&A, 265, 115 